\title{
A Study of Computerized Techniques Used in Ultrasound Breast Cancer Detection
}

\author{
V. Mary Kiruba Rani \\ Research Scholar, \\ Dept of Computer Science and Engineering, \\ Alagappa University
}

\author{
S.S. Dhenakaren, $\mathrm{PhD}$ \\ Professor, \\ Dept of Computer Science and Engineering, \\ Alagappa University
}

\begin{abstract}
Staging of cancer is said to be unsigned in natural history. In this advanced growth of medical research, finding out the tumor growth is demanding in the midst of the researchers. The aim of this study is to provide a pathway for predicting tumor stages with the help of processing tools. Since breast cancer tumor is said to in the worlds second place of disease, prevention of higher growth sound be maintained. With sticking out to reduce costs and high predicted upshot ultrasound images, were chosen. Ultrasound screening images are targeted to produce an ending over currently predicting techniques. Breast cancer tumor growth findings lead to the public to get aware of frequent possible medical checkup. Overcoming the margins of existing techniques and methods, a new approach is stretched out for prediction phase. Neural networks and image segmentation based concepts are composed and a final wrapping up will lead advanced finding of efficiency. This study starts from the collection of ultrasound images, followed by the methods that define for predicting the cancer tumor, and the limitations to be met.
\end{abstract}

\section{Keywords}

Study, ultrasound, breast cancer, neural network, image processing.

\section{INTRODUCTION}

In India, breast cancer is in the leading cause of death and also one of the reasons for death is lacking early stages of cancer. Even though more awareness program is carried out, early finding of cancer is not yet found. Also, the growing period of cancer tumor is not being processed. The concept which was chosen for this research will meet the growing period. A bunch of cancers is spread over the area, but the main reason why choosing breast cancer is: over $14.3 \%$ of people were affected with breast cancer. Fast retrieval of cancer tumor prediction is taken over with MRI and CT scan images. But the aim of this research is with the use of ultrasound image, how the growth of breast cancer tumor can be graded. The main issue is that ultrasound images are said to be no clear images, then how can it be solved? Whether a tumor is said to be cancerous or noncancerous and what attributes reflects over prediction of cancer? What limitations are not yet met over recent research methodology?

To come back with for all above queries, a brief study is focused on for findings. Ultrasound screening is the initial testing stage and the images in this screening will not be easily predictable. Thus, computerized techniques are used for predicting tumors. In recent years, a few proposed works have gone through and various techniques are implemented. Image processing using MATLAB plays the vital role in medical image prediction. The Tumor Node Metastases (TNM) system is said to be the backbone of cancer staging. It involves in predicting how much of cancer and where the tumor is located. Based on the size stages are marked as IA, IB, IIA, IIIA, IIIB,
IIIC, and IV. This concept is involved in every tumor extraction process [2]. Various image processing techniques like noise removal, classification, segmentation clearly help to predict the cancer tumor. One of the most obvious problems was finding the cancer tumor also its growth at an early stage. The techniques involved in this research have a possible approach to get an accurate result. Breast cancer is also tested in the same way.

Finding the characteristic features of a large cancer tumor image is easy whereas in the small images, primary malignancy features are not found in many but it is active as cancerous using its secondary effects. So the research starts from the stage when it shows the cancer characters.

The neural network [3] in image processing will come up with easy learning of cancer tumor, such that the center of attention is on the attributes like height, width, margin, adjacent nodes. Also, image segmentation process is put into practice for separating the cancer tumor from an image. An automatic prediction of growth in cancer tumor of a single person is welcomed by the public. Thus, the techniques are studied to get a better result over the objective.

\section{ANALYSIS OF METHODS}

Nowadays, diseases are next to people of any age group. Even though the environment and the food items are the primary reason for boosting the diseases, the symptoms and the characteristic features of any disease is learned well and by producing a new approach can prevent that disease from future growth. This analysis is for breast cancer research. The methodology used for predicting cancer tumor is basically image processing techniques. Also, the neural network concept is implemented in learning the process. This research is carried out with the images collected from ultrasound screening machine and from it a prediction of various cancer stages is held in.

\subsection{Attributes in ultrasound cancer tumor}

Certain symptoms in an image confirm the appearance of cancer. The characteristic of breast cancer tumor used in research findings is size, height, width, adjacent nodes, calcification, lesion, location, age, tissue type, date of finding, date of treatment. Along with this, physical examination results are combined with to determine the stage.

\subsection{Spatial classification of tumor}

The classification [4] of the tumor is carried out with TNM (Tumor, Node, and Metastasis) [5] system. This process includes: T- based on the size of the primary tumor and whether it has grown with nearby tissues. Node $\mathrm{N}$ - indicates whether the cancer has spread to the nearby lymph nodes and finally Metastasis, M- point out whether the cancer spread to a distinct part. This is the basic key point over cancer tumor and currently implemented to tumor prediction with CT and MRI scanning process. But it is a lack in consequence of this model 
with ultrasound screening (US) since; the US is low-density image screening. The classified output will be useful for the new patients of the same characteristics.

\subsection{Shape representation}

The tumor is predicted based on its shape. If the shape is in irregular or well-defined form, then it is said to be cancer tumor. Also, this process is said to be tedious because Ultrasound Screening gives different structures like connective tissues, nodes, etc. This process is done by tumor shape segmentation using Self Organizing Map (SOM) [10]. This gives the evolution of segmentation by artificial neural network (ANN) SOM to the automatic detection of nodules contour in regions of interest (ROIs) from clinical images of breast ultrasound. [6]

\subsection{Multiview Tumor Detection}

A linear regression model is estimated for finding out the lesions from tumor candidates. Aiming to get better result normal attributes is used for detecting cancer [11]. A candidate's higher tumor likelihood is examined as a lesion. In Multiview tumor cancer detection, since likelihood tumor sizes are premeditated, some features have less information, thus backward feature elimination is carried out. A training model is developed and used to examine the testing test.

A statistical significance of feature based tumors is grouped into several types. Low classification tumor group is removed from the feature set because of low feature information. The grouped training model is repeated till all the groups are evaluated and finally tumor detection in all orthogonal views is combined together to find the accuracy. Since in ultrasound screening the images will be of different sections, it should be combined into one for easier computational purpose. Multiview cancer detection is an interactive region for the tumor contender which focus better for tumor growth phase. [7]

\subsection{Segmentation of lesion}

Segmenting a tumor image is the ultimate task of cancer research. Ultrasound images are said to be unclear, thus all the speckles are detected initially before processing using filtering techniques. Edges in an image are segmented using an unmasked filtering algorithm. The lesion predicted is then classified based on its density value. The lesion is resulted in being malignant [8]

Since, ultrasound screening is the initial scanning device, but the output of a scanned image will not be predictably good. Thus, the radiologist would refer to tests like the biopsy, pathology test, and physical test if the scanned image is in the form of an assumption.

Even though few methodologies are discussed, a row of limitations should meet. They are,

- Accurate prediction of lesions without moving to testing.

- A user interface system should be developed so that without interference of radiologist, the person who scans can itself predict the disease.

- $\quad$ Same person's details should be maintained so that a classification can be obtained. With the classified output, a new patient who comes can control the further growth of the tumor.

- Location of tumor growth should be identified.

- Tumor stage should be predicted

\section{STATISTICAL INFORMATION FOR CANCER RESEARCH}

Cancer patients are growing day by day. In India, for the year 2012: 144,937 women were newly detected with breast cancer. 70,218 women died of breast cancer.144937/ $70218=2.06=$ round it off to 2 . So rough, in India, for every 2 women newly diagnosed with breast cancer, one lady is dying of it.

World Health Organization (WHO) declared a report earlier that for the year 2015, there will be an estimated 1, 55,000 new cases of breast cancer and about 76000 women in India are likely to die of the syndrome. The gap only seems to make wider, which means, need to work aggressively on early detection. Breast cancer is most common among all over India and it accounts for $25 \%$ to $31 \%$ of all types of cancers in women particularly in India.

The one and only way of cancer treatment is to reduce the growth of tumor without spreading to nearby cells. Thus a computerized approach for used techniques and its limitation over current era is explained.

Ultrasound screening methodology proves to be a standardized process when the accuracy for finding any prediction is good. The non-clear images are filtered with techniques like preprocessing, smoothening, removal of noise and the final output is a clear image used for computation.

The measurement techniques over ultrasound existing methods are justified below. Table 3.1 gives the filtered value for measuring breast cancer of an ultrasound screened image. It includes calculating Specificity, Sensitivity, Positive predicted value, Negative predicted value and overall accuracy of all measurements.

Table 3.1 Analysis for breast cancer [9]

\begin{tabular}{|l|l|l|l|l|l|}
\hline $\begin{array}{l}\text { Measur } \\
\text { ement }\end{array}$ & $\begin{array}{l}\text { Accur } \\
\text { acy } \\
(\%)\end{array}$ & $\begin{array}{l}\text { Specif } \\
\text { icity } \\
(\%)\end{array}$ & $\begin{array}{l}\text { Sensitiv } \\
\text { ity } \\
(\%)\end{array}$ & $\begin{array}{l}\text { Predicted } \\
\text { positive } \\
\text { value } \\
(\%)\end{array}$ & $\begin{array}{l}\text { Predicted } \\
\text { negative } \\
\text { value } \\
(\%)\end{array}$ \\
\hline Breast & 94.25 & 91.67 & 96.08 & 94.29 & 94.23 \\
\hline
\end{tabular}

Above table describes that the accuracy in predicting the features in an image is about $94.25 \%, 91.67 \%$ of Specificity, $96.08 \%$ of sensitivity, $94.29 \%$ of the positive predicted value and $94.23 \%$ of Negative predicted value.

Table 3.2 Accuracy Table for some Standard Techniques for Tumor Prediction

\begin{tabular}{|c|c|}
\hline Technique/Methods & Accuracy \\
\hline & \\
SVM Classifier & $98 \%$ \\
Morphology \& 3D Analysis & $96 \%$ \\
PCA & $88 \%$ \\
\hline
\end{tabular}


Table 3.3 Comparison of different Techniques used in Ultrasound Screening

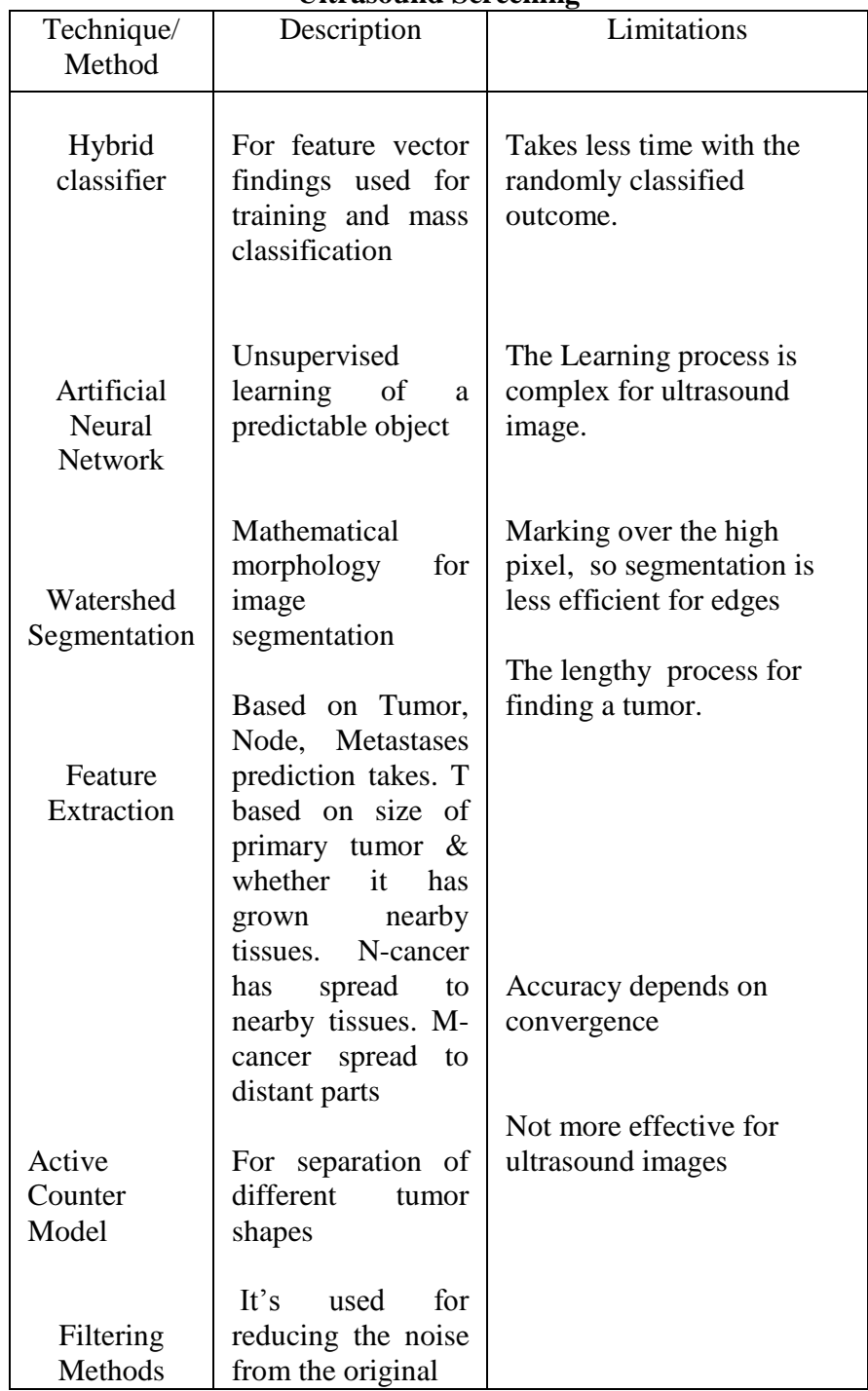

Various techniques applied for getting the features in an ultrasound image. The used techniques predict few limitations over finding features. Thus a new technique Tumor Growth Model (TGM), gives better accuracy and triumph over the limitations of existing methods.

\section{PROPOSED SCENARIO FOR SEGMENTING TUMOR}

Observing the limitations on various existing methods, a new model can be framed for better processing. Pointing to the current use of cancer research, a new approach is developed for determining the tumor growth. Ultrasound images are passed as input, and a clean, noise removal technique is added for better quality.

It can be done by converting the image to RGB and again converting back into grayscale. Now using this image, dispensation gets start. The pixel values with low and high values are found together with histogram analysis. Edges are detected and segmentation process is carried for separating the tumor from an image. The difference between benign and malignant or initially learned computerized.

The proposed model named as Tumor Growth Model (TGM) enters with checking whether the given image is an ultrasound image or not. Histogram representation of an image confirms the source of the image. If the image is valid, then an ultrasound image is given as the input. The process starts with removing the unclear formats of an image and find out the edges of high intensity values. The values which are similar to the high threshold values are clustered.The following diagram explains the functionality of the proposed system,

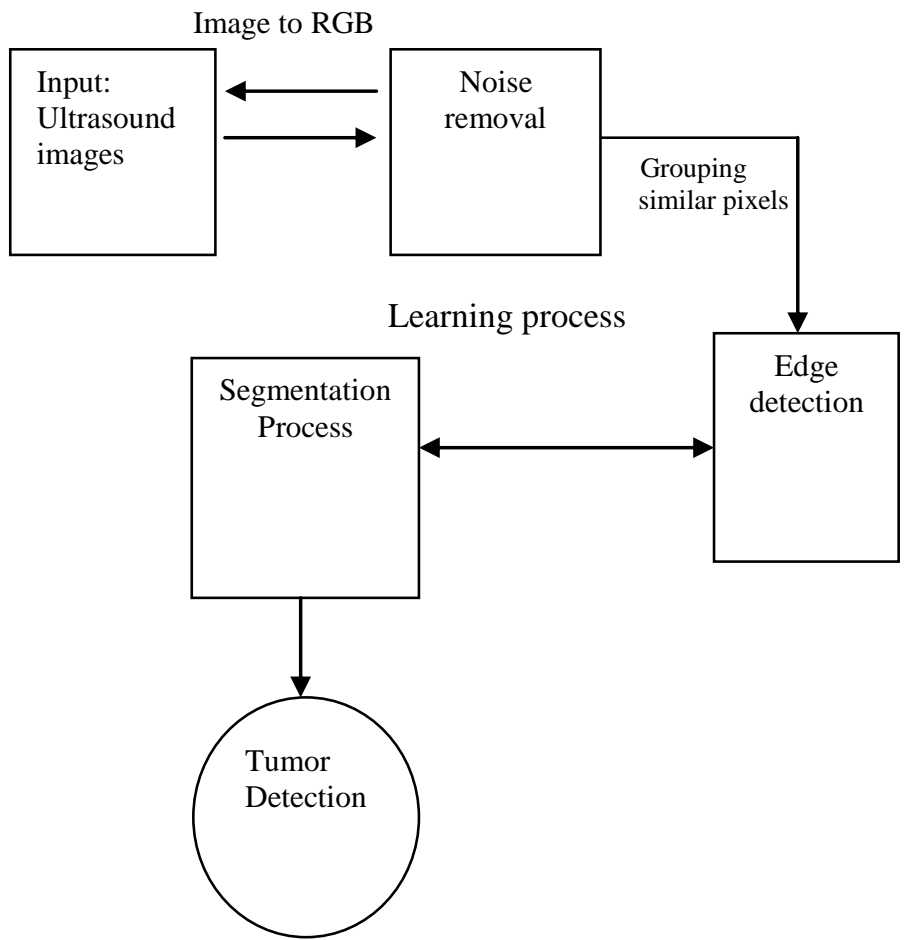

Figure 4.1 Model for tumor segmentation in ultrasound image

The weight vector should be updated regularly until the convergence occurs. The main objective of the SOM is to produce a neighbor in input space projecting towards neighborhood in output space. Completing the learning process will provide a tool to classify a tumor from an image. Finding out the cancerous tumor of an ultrasound breast image steps into new areas for tumor growth prediction. Finally, separation of the tumor gets out.

An algorithm for proposed methodology Tumor Growth Model (TGM) explains how to find out the tumor growth stage of a single person.

\section{Proposed Algorithm TGM ()}

\section{\{}

1. Let $\mathrm{k}$ be input ultrasound segmented image.

2. Calculate total pixel values.

3. Mark the location of the cancer and fix a pixel fixed point.

4. Calculate the distance between the center and to the edge.

5. Growth $=\sum_{0}^{n}(k$, tumor size $)$

6. Group the result with individual patient details.

7. Result=(age, date) $\triangleright \backslash$ (growth)

8. Growth prediction for an individual patient is obtained 
The proposed TGM algorithm is implemented for the tumor growth model by accessing the segmented tumor image. The segmentation is based on threshold value. The segmented image is then stored into the database. The database consists data format of image along with old calculated cancer images of a person with different cancer stage. Calculating the pixel values of the segmented image, find the midpoint of it and trace Euclidean distance. Pick up the distance and compare with other details such as age, blood group of the same person. Growth of a person is predicted by finding the difference between the growth of tumor on various stages along with the age and place of particular person. Thus, the growth of cancer tumor within a certain period for an individual person is predicted.

\section{CONCLUSION}

The study has overviewed the techniques and methodologies for ultrasound screening image of breast cancer. From the study, it is observed that there are limitations over existing methods such as obtaining very tiny feature, to predict the cancerous of a person; automatic finding of an image is benign or malignant. These limitations are considered and becoming a challenging task to TGM model. This model has required a high quality image of ultrasound image for cancer detection. So, as a first step, a high quality image is generated, segmented and then the limitations of earlier methods are invoked. Thus, the study is helped to get a positive outcome for classification.

\section{REFERENCES}

[1] Dr.Agarwal's Center for Tanslational Research, Tirunelveli.

[2] K.Subashini, K.Jeyanthi, "Breast Cancer Detection and Classification in High Resolution Ultrasound Images", international journal of innovative research in electrical, electronics, instrumentation and control engineering Vol. 2, issue 5, May 2014.

[3] Karem Daiane Marcomini and Homero Schiabel, "Nodules segmentation in breast Ultrasound using the artificial neural network self organizing map",
Proceedings of the World Congress on engineering 2012 Vol II WCE 2012, July 4 - 6, 2012, London, U.K.

[4] Pooja Kamavisdar, Sonam Saluja, Sonu Agrawal, "A Survey on Image Classification Approaches and Techniques", International Journal of Advanced Research in Computer and Communication Engineering Vol. 2, Issue 1, January 2013.

[5] American joint committee on cancer, $7^{\text {th }}$ edition.

[6] Bipul Pandey, Tarun Jain , Vishal Kothari and Tarush Grover, "Evolutionary Modular Neural Network Approach for Breast Cancer Diagnosis “ , IJCSI International Journal of Computer Science Issues, Vol. 9(1), January 2012

[7] Chiao Lo, Yi-Wei Shen, Chiun-Sheng Huang and RueyFeng Chang, "Computer-Aided Multiview Tumor Detection for Automated Whole Breast Ultrasound", Ultrasonic Imaging 2014, Vol 36(1) 3-17.

[8] Miguel Alem'an-Flores, Patricia Alem'an-Flores, Luis ' Alvarez-Le'on, Rafael Fuentes-Pav'on, Jos'e M. SantanaMontesdeoca, Filtering, Segmentation and Feature Extraction in Ultrasound Evaluation of Breast Lesions.

[9] Y.Hunang, K.Wang, B.Chen,'Diagnosis of breast tumors with ultrasonic texture analysis using support vector machines", neural computing and applications, 2006, vol15(2 164-169.

[10] Juha Vesanto, Johan Himberg, Esa Alhoniemi and Juha Parhankangas," Self-organizing map in Matlab: the SOM Toolbox", proceedings of the Matlab DSP conference, ESPOO, finland, 1999,pg- 35-40

[11] Valea FL, Katz VL," Breast diseases: diagnosis and treatment of benign and malignant disease" Comprehensive Gynecology. 5th ed. Philadelphia, Pa: Mosby Elsevier; 2007. p. 15. 